\title{
3D Manipulation and Imaging of Plant Cells using Acoustically Activated Microbubbles
}

\section{Journal Article}

\section{Author(s):}

Läubli, Nino (iD); Shamsudhin, Naveen; Vogler, Hannes; Munglani, Gautam; Grossniklaus, Ueli; Ahmed, Daniel; Nelson, Bradley (D)

Publication date:

2019-03-13

Permanent link:

https://doi.org/10.3929/ethz-b-000325696

Rights / license:

In Copyright - Non-Commercial Use Permitted

Originally published in:

Small Methods, https://doi.org/10.1002/smtd.201800527

Funding acknowledgement:

166110 - Mechanical Basis for the Convergent Evolution of Sensory Hairs in Animals and Plants (SNF) 
This is the accepted version of the following article:

N. F. Läubli, N. Shamsudhin, H. Vogler, G. Munglani, U. Grossniklaus, D. Ahmed, B. J. Nelson, Small Methods 2019, 1800527,

which has been published in final form at https://doi.org/10.1002/smtd.201800527

\section{D Manipulation and Imaging of Plant Cells using Acoustically Activated Microbubbles}

Nino F. Läubli, Naveen Shamsudhin, Hannes Vogler, Gautam Munglani, Ueli Grossniklaus, Daniel Ahmed*, Bradley J. Nelson

N. F. Läubli, Dr. N. Shamsudhin, Dr. D. Ahmed, Prof. B. J. Nelson

Institute of Robotics and Intelligent Systems, ETH Zurich, Tannenstrasse 3, CH-8092 Zurich, Switzerland

E-mail: dahmed@ethz.ch

Dr. H. Vogler, Dr. G. Munglani, Prof. U. Grossniklaus

Department of Plant and Microbial Biology \& Zurich-Basel Plant Science Center, University of Zurich, Zollikerstrasse 107, CH-8008 Zurich, Switzerland

Keywords: acoustics, bubbles, lab-on-a-chip, plant cells, 3D reconstruction

The precise manipulation of single cells and organisms opens exciting new possibilities for biological research. In this article, we demonstrate an acoustic rotational manipulation method for imaging single cells of different plant species (pollen grains of Lilium longiflorum and Arabidopsis thaliana). Acoustically activated microbubbles generate radiation forces as well as microvortices in the aqueous medium, which allow various specimens to be trapped and precisely rotated. We studied the rotational behavior of individual plant cells and controlled their motion to facilitate 3D fluorescent microscopy. We demonstrated the use of this manipulation technique for high-resolution 3D optical reconstructions of non-transparent samples. We further demonstrated the applicability of this method for open-microchannel arrangement, which may enable multiplexed 3D access to samples for microsurgery and injection.

\section{Introduction}

Development and morphogenesis of biological structures is governed by spatiotemporal modulation of biochemical and mechanical properties across single cells, tissues or 
organisms. Consequently, their investigation requires a precise control of the specimen's position and orientation. To date, numerous methods and devices have been developed to perform two- or three-dimensional (3D) manipulation of single cells, organisms, or microparticles by utilizing hydrodynamic ${ }^{[1]}$, electric ${ }^{[2]}$, magnetic ${ }^{[3,4]}$ or optical $^{[5]}$ techniques. $^{2}$ However, many of the existing methods require sophisticated instrumentation or particular specimen properties, significantly limiting their manipulation capabilities for biological samples.

Acoustics provide a noninvasive and robust transduction mechanism that has been widely used in micromixing ${ }^{[6-8]}$, micropumping ${ }^{[9]}$, particle or cell separation ${ }^{[10,11]}$ and manipulation $^{[12-15]}$, protein crystal patterning ${ }^{[16]}$, switches ${ }^{[17]}$, droplet production ${ }^{[18,19]}$, drug delivery $^{[20]}$, and actuation of micro- and nanoswimmers ${ }^{[21-23]}$. Acoustic wave induced microvortices around solid structures and bubbles have been used to perform manipulation of small objects. ${ }^{[24-26]}$ The excitation of trapped air bubbles has been applied to perform translational and rotational manipulation of single animal cells and animal model organisms such as Caenorhabditis elegans. ${ }^{[27]}$ Though microfluidic and acoustofluidic technologies have been widely used in animal models at the cellular, tissue and organ level, there has been limited availability of such tools in plant biological research. Single plant cell phenotyping tools are crucial for advancing plant biological research considering that plants have an important function in the food chain and economic systems.

Plant cells, such as the pollen grains ${ }^{[28-30]}$ and pollen tubes ${ }^{[31-33]}$, play a key role in plant sexual reproduction, and serve as model systems for cellular morphogenesis. Pollen, also known as the male gametophyte of flowering plants, carries and transports the two spermcells within its cytoplasm. Pollen is produced in the anthers of the flower, and is carried by wind, insects, birds or other pollinators to a receptive stigma of the same or another flower (Figure 
$\mathrm{S} 1)$. During this process, pollen grains have the crucial role of protecting the sperm cells as well as the vegetative nucleus, which controls pollen tube behavior during pollination and fertilization. ${ }^{[34]}$ The pollen surface consists of two major regions: the mechanically stiff exine, a structure that covers most of the cell wall, and the colpi, a mechanically softer region where the intine layer of the cell wall is exposed. These mechanically soft regions are of special interest, since they enable the pollen grain to fold and unfold during dehydration and rehydration, respectively. ${ }^{[35]}$ After reaching a receptive stigma, the pollen grain rehydrates and unfolds, and soon after a tubular extension called the pollen tube is generated by anisotropic expansion of the pollen grain. The exact region on the pollen grain from which the pollen tube emerges differs among species. Pollen grains are of great interest for 3D micromanipulation as they have a complex geometry and a variated surface structure, which renders $2 \mathrm{D}$ manipulations ineffective. Additionally, sub-cellular optical characterization is difficult as these cells possess a thick cell wall, which renders them opaque, and their optical phenotyping would greatly benefit from a system that provides a combination of $3 \mathrm{D}$ rotation and imaging.

This article demonstrates precise in- and out-of-plane 3D rotation of Lilium longiflorum (lily) and Arabidopsis thaliana pollen grains using acoustically activated microbubbles in both closed- and open-microchannel arrangements. Although the dimensions of these two species of pollen grains are different, a single microbubble size was successfully used for all manipulations. The rotational behavior of these non-spherical plant cells was characterized at angular velocities up to 3,000 r.p.m. and their heterogeneous surface morphologies were visually analyzed. A high-resolution 3D reconstruction of a fluorescently labeled pollen is performed and we highlight the advantages of this technique to other imaging methods. Furthermore, the open-microchannel arrangement provides a complete access to the surface of the specimen, which can enable exciting future research possibilities such as microinjection and 3D stiffness mapping through micro-indentations. 


\section{Results and Discussion}

\subsection{Cell Rotation in Closed Microchannels}

We use acoustic waves to manipulate pollen grains. When liquid, such as water, is injected into the microchannel (Figure 1 a), Figure S2), microbubbles get trapped within the microcavities. ${ }^{[36]}$ The microbubbles were found to be stable for at least 30 minutes, and no significant difference was observed between the use of de-ionized water or growth medium (Figure S3). When an acoustic field in the kilohertz range is generated by the piezo-electric transducer, the bubbles start to oscillate. The oscillation amplitude becomes maximum when the bubbles are driven at their resonances. The microbubble oscillations lead to large velocity gradients close to the air/liquid interface, which eventually results in a pair of counter-rotating vortices $^{[37-39]}$ as shown in Figure 1 b) (Movie S1). Depending on the excitation frequencies, multiple or higher modes of the bubble oscillation are achieved, resulting in different vortex shapes. Figure $1 \mathrm{c}$ ) shows a $10 \mu \mathrm{m}$ polystyrene particle following an out-of-plane vortex. The image sequence in Figure 1 d) visualizes the movement of the microbead as it changes in and out of the focal plane. This is represented by the significant change of the area marked with the magenta arrow. The area gets smaller as the bead gets more into focus plane while its size increases when it moves out of focus (Movie S2). We take advantage of these vortices developed near the oscillating microbubbles to perform high throughput controlled in- and out-of-plane rotations of single plant cells.

We present the rotational capabilities of the microfluidic chip using lily pollen grains. Rehydrated pollen grains are injected into the microchannel along with growth medium, simultaneously enclosing air bubbles in the microcavities. By tuning to the appropriate frequency of the acoustic waves, the grains are first trapped near the excited bubbles due to radiation forces. Figure 2 a) shows the in-plane rotation of a lily pollen grain (Movie S3). Stable rotation is generated by bubble oscillation, and the rotation rate can be controlled by 
the applied voltage of the piezo-electric transducer while maintaining a constant excitation frequency. The excitation frequency is manually tuned to alter the flow field around the bubble, resulting in an out-of-plane rotation of the pollen grain (Figure 2 b), Movie S4). Although the pollen grain rotates around a fixed axis, the rotation axis itself undergoes a small precession. This can be attributed to the shape and composition asymmetry of the pollen grain. To better understand the 3D motion of the pollen grain, the graphic insets show the rotation when observed along the rotational axis, i.e. in the direction of the black arrow. The highlighted conically shaped part represents the position of the colpus, also marked in the actual image sequence, by red arrows. The rotational axis for the out-of-plane manipulation is mainly imposed by the geometrical anisotropy of the plant specimen, however, additional effects may be caused by surface roughness or weight distribution and require further investigation.

Next, we demonstrate the rotation of Arabidopsis thaliana pollen grains, which are much smaller and more spherical than those of lily. Although these grains are approximately 4 to 5 times smaller than lily pollen grains, ${ }^{[31]}$ the same device geometry can be utilized for their 3D rotational manipulation. Figure 3 a) shows a single pollen grain rotating in-plane (Movie S5). The image sequence in Figure 3 b) (Movie S6) shows the stable out-of-plane rotation of the Arabidopsis pollen grain.

It is observed that, while the shape of the vortex is defined by the driving frequency, ${ }^{[40]}$ the voltage applied to the transducer influences its strength and, therefore, the angular velocity of the rotating plant cell. The in-plane rotations of individual plant cells are characterized and plotted separately for lily (blue) as well as Arabidopsis (red) pollen grains in Figure 4. Although the in-plane rotation is less relevant for three-dimensional imaging compared to outplane rotation, it is crucial for mechanical interventions, such as microinjection, where the 
specimen is accessed horizontally inside a lab-on-chip device. ${ }^{[41]}$ Figure 4 a) and 4 b) demonstrate that Arabidopsis pollen grains present a more linear relation between the rotation angle and the time than that of the larger lily pollen grain. A reason might be the stronger shape anisotropy of the lily pollen grain, leading to a change of the area directly exposed to the strong vortex field near the bubble surface. Nevertheless, other factors might influence the rotation, such as the relation of the grain size to the bubble size, distribution of density of the pollen grains or acceleration of cytoplasm inside the pollen grain.

Figure $4 \mathrm{c}$ ) and $4 \mathrm{~d}$ ) illustrate the tunability of rotational velocity of the specimen. The rotational velocities (r.p.m) of lily (blue) and Arabidopsis (red) pollen grains with respect to changing voltage at fixed excitation frequencies. The angular velocity increases with the applied voltage (Movie S7). While for lily pollen grain it is calculated that the voltage influences the angular velocity by the power of 1.53 , the Arabidopsis pollen grains show a dependency of the angular velocity to the driving voltage corresponding to the power of 2.49 . This is also shown by the exponential fits in the upper left corner of Figure $4 \mathrm{c}$ ) and d). The large difference between the plant cells can be explained by instabilities observed in the rotation of lily pollen grains at low voltages. At low applied power, the lily pollen decelerates due to friction between the cell wall and the bottom of the microchannel. For high voltages, this effect is reduced, since friction plays a secondary role compared to the large forces from acoustic streaming.

The rotational velocity of the specimen can be manipulated by the voltage applied and the acoustic excitation frequency. Other factors that can contribute to the strength of the rotational manipulation include distance from the microchannel to the piezo-transducer, size of the microbubbles, and attenuation of the acoustic waves in polydimethylsiloxane (PDMS) ${ }^{[42]}{ }_{-}$ based microchannels. 


\subsection{Principle of Rotational Manipulation}

An important advantage of our system is that micro-objects (i.e., single pollen grains) are trapped by the oscillating microbubbles and their 3D rotational motion can be precisely controlled by the acoustic waves. The rotational behavior of the specimen depends on the acoustic torque acting on the specimen, which is created by the acoustic microstreaming of the bubble oscillation.

At low Reynolds number, the acoustic torque balances the viscous torque.

The Reynolds number $(R)$ of the acoustic microstreaming near an oscillating microbubble can be calculated as follows: ${ }^{[43]}$

$R=\varepsilon^{2}\left(\frac{2 \pi f a^{2}}{v}\right)^{1 / 2} \approx 0.1$

where $\varepsilon \sim 0.1$ is the ratio between the oscillation amplitude and the radius of the microbubble, $a \sim 30 \mu \mathrm{m}$ is the radius of the microbubble, $f=20 \mathrm{kHz}$ is the oscillation frequency, and $v \sim 1.0 \cdot 10^{-6} \frac{\mathrm{m}^{2}}{\mathrm{~s}}$ is the kinematic viscosity of the growth medium used in the experiments. Assuming that the specimen is spherical, the viscous torque $\left(\tau_{V}\right)$ with the Stokes drag acting on a spherical object can be approximated: ${ }^{[44]}$

$\tau_{V}=8 \pi \mu a_{S}^{3} \omega_{S}$

in which $\mu, a_{S}$, and $\omega_{S}$ are the dynamic viscosity of the surrounding medium, the radius of the spherical object, and the angular velocity, respectively. The acoustic torque $\left(\tau_{A}\right)$ acting on the specimen comes from two major forces: acoustic streaming and radiation acting onto the object due to the oscillating bubble. The acoustic torque can be described by the following relationship: ${ }^{[21]}$

$\tau_{A} \propto P^{2} \propto V^{2}$

where $P$ and $V$ are the pressure amplitude and the voltage applied to the transducer, respectively. The acoustic torque has to exactly balance the viscous torque to achieve stable 
rotational motion of the specimen. Therefore, we obtain a relationship for the angular velocity

$\omega:$

$\omega \propto V^{2}$

which demonstrates a quadratic dependence of the angular velocity of the rotating specimen on the voltage applied to the exciting piezo-electric transducer, which agrees reasonably well with the experimental data presented above.

\subsection{Fluorescent Imaging}

Fluorescent imaging allows for both the observation of surface morphology and the cellular composition inside the sample. Our acoustofluidic device offers direct integration into a fluorescence microscope. The experiments demonstrate controlled tuning of the specimen's angular velocity from 0 to 3000 r.p.m by using moderate excitation voltages $\left(1-15 \mathrm{~V}_{\mathrm{PP}}\right)$. The exact orientation and position of a component of interest within the cell can be determined at low rotational speeds. In addition, reorientation can be used to ensure a direct view of intracellular features, avoiding obstruction by auto-fluorescence of neighboring parts.

The nuclei of the lily pollen grains shown in Figure $\mathbf{5}$ a) and b) (red circle at 1.8 seconds in Figure 5 b)) are labeled using 4',6-diamidino-2-phenylindole (DAPI), a fluorescent dye that binds nucleic acids, i.e., DNA. Figure 5 a) (Movie S8) demonstrates a slow in-plane rotation of a fluorescently labeled lily pollen grain. The nucleus retains the same relative position with respect to the cell during its rotation. A stable out-of-plane rotation is presented in Figure $5 \mathrm{~b}$ ) (Movie S9). The small bright spot is the vegetative nucleus of the bicellular lily pollen grain, while its generative cell, the precursor of the two sperm cells, can only be seen through the auto-fluorescent colpus (highlighted with a red circle at 1.8 seconds). Fluorescent imaging could be further improved by stepwise rotation of the sample through pulsed actuation of the piezo-transducer. The Reynolds number of an oscillating bubble in an acoustic field was 
estimated to be less than 1; thus, viscous forces dominate inertial effects. The rotating specimen comes to a stop instantaneously by switching off the acoustic field (Movie S10). This enables longer exposure times at regions of interest. Our system shows clear advantages over previously developed plant cell monitoring microfluidic devices by offering increased optical access and visibility through the re-orientation of the cell. ${ }^{[45,46]}$

\subsection{Optical 3D Reconstruction}

3D reconstruction of single cells or microorganisms could enable better understanding of their bulk structures and surface morphologies. It can be used as a powerful tool to investigate their interaction with the environment while providing new structural insight. The creation of optical 3D reconstructions requires high-resolution imaging and precise manipulation methods. Additionally, if fluorescence microscopy is used, the sample must be rotated slowly to prevent blurriness.

Figure 5 c) shows a dense 3D reconstruction of a fluorescently labeled lily pollen grain. Using time-lapse imaging, we captured several rotations of the pollen grain at multiple focal planes due to the ellipsoidal (non-symmetric) shape of the grain. The reconstruction was generated from the image stacks using a combination of image filtering and the Structure from Motion (SFM) technique (see Experimental Section). The dense, high-resolution 3D reconstruction provided a well resolved pollen grain exine structure in the equatorial region between polar angles (along the long axis of the grain) of approximately 50 and 130 degrees. The poles of the pollen grain were not reconstructed as the rotational axis prevented them from being in focus at the given camera angle. This method shows several advantages over other devices, such as 3D imaging achieved by hydrodynamic rotation along a microchannel. ${ }^{[1]}$ Our method enables precise control of the angular velocity of the sample and the ability to start and stop the rotation instantaneously, which can reduce the motion-blur. ${ }^{[47]}$ As the observed sample is 
trapped near the microbubble interface and stays in focus, high-resolution optics can be used. Additionally, as the angular velocity can be tuned via an arbitrary electronic function generator, no expensive micro-pump system is required. This method enables a low-cost option to reconstruct non-transparent samples, where the use of confocal microscopy is limited. Furthermore, this approach does not require any surface modifications, which might alter the biochemical surface structure of the specimen. Finally, as no pressure gradient or flow has to be developed along a microchannel, it can also be used in a setup with an openmicrochannel.

Also, compared to the well-established imaging method such as the scanning electron microscopy, ${ }^{[48]}$ our acoustic manipulation method provides a fast and inexpensive approach to detect biological changes of the surface morphologies of living pollen grains without complex sample preparation ${ }^{[49]}$ or the use of a vacuum, thus, enabling plant cells to be suspended in growth medium. The $3 \mathrm{D}$ reconstruction could be further improved by using a calibrated optical setup. Additionally, optimizing the fluorescence protocol might reduce the strong feedback of the colpus, which can prevent blurriness in its immediate surrounding, thereby increasing the azimuthal angle for reconstruction.

\subsection{Cell Rotation in Open Microchannels}

The 3D rotation of micro-objects in closed microchannels provides a simple and non-invasive method to study the surface morphology of individual plant cells. The closed microchannel design enforces a limitation to optical feedback and, thereby, complicates the thorough investigation of the specimen. Here we demonstrate the rotation of pollen grains in open channels, which additionally provides access for micro-mechanical interrogation of the sample by integration with micro-force sensing systems, ${ }^{[50,51]}$ such as the cellular force microscope, or for micro-injection of high molecular weight dyes or DNA, which are cell wall 
or membrane impermeable. ${ }^{[52,53]}$ The open-microchannel arrangement is developed by bonding the PDMS microchannel upside-down onto a glass slide, as shown in Figure 6 and Figure S2. Submerged lily pollen grains are placed dropwise onto the microchannel, simultaneously trapping air bubbles in the microcavities due to the hydrophobic surface properties of the PDMS. During this process, the transducer is excited. This allows the pollen grains to sink, where they become trapped in the microstreaming vortices of the oscillating microbubbles. The PDMS device is surrounded by Plexiglas to prevent leakage, while the top remains open for direct access to the sample. Our device containing two parallel microchannels is shown in Figure $6 \mathrm{~b}$ ). Parallelizing entire channels allows for multiplexed observations of the pollen grains. Several lily pollen grains are trapped by the microbubbles' oscillations and are rotating around different axes, depending on the size and shape of the microbubbles and, therefore, their modes of excitation. The vortices above the oscillating microbubbles did not interfere with the specimen's rotation at the side vortices. The top vortices could provide more trapping possibilities at different planes, especially for the openmicrochannel arrangement, as shown in Figure S4. Additionally, the developed vortices could assist in attracting and navigating sinking pollen grains (due to gravity) near the microbubbles

In a closed-microchannel arrangement, a microbubble grows over time, which shift its resonance frequency (Figure S5). This shift alters the strength of the generated microstreaming and subsequently deviates the angular velocity of the manipulated specimen (Figure S6). According to equation (5) for a free-standing air bubble, an increase in the size leads to a decrease of the bubble's resonance frequency: ${ }^{[54]}$

$f=\frac{1}{2 \pi R}\left(\frac{3 p_{0}}{\rho_{l}}\right)^{1 / 2}$

where $f, R, p_{0}$, and $\rho_{l}$ are the resonance frequency, the radius of the microbubble, the ambient pressure and the density of the liquid, respectively. For the closed-microchannel 
arrangement, this effect can be reduced by increasing the pressure inside the channel by injecting liquid. The opposite behavior of the microbubble stability is observed for open microchannel, i.e., the microbubble tends to shrink. According to the equation (5), this leads to an increase of the resonance frequency. This phenomenon can be counteracted by acoustically activating the microbubble leading to the growth of the microbubble by a process known as rectified diffusion. ${ }^{[55]}$

The stable out-of-plane rotation of a lily pollen grain trapped by a microbubble is shown in the image sequence of Figure $6 \mathrm{c}$ ) (Movie S11). Although the acoustic waves attenuate when they travel from the glass slide through PDMS and to the microbubbles, we report that a single-digit peak-to-peak voltage is sufficient to produce stable rotation. The required voltage can be further minimized by optimizing the position of the transducer, i.e., reducing the distance between the transducer and the PDMS structure, as well as reducing the thickness of the PDMS device. Manipulation utilizing low power is advantageous since the heat dissipation will not damage any biological specimen.

\section{Conclusion}

The accurate positioning and rotational manipulation of plant cells in $3 \mathrm{D}$ provides a powerful tool for optical interrogation of the specimen. We have demonstrated the successful use of acoustically activated microbubbles to trap non-spherical plant cells of varying sizes, enabling the study of their complete surface morphology by controlled in-plane and out-of-plane rotations. The acoustically activated microbubbles allows for pulsed and continuous mode rotations with precise control, without any sample damage. The rotational performance of two important plant cell models were evaluated. We presented its use for 3D optical reconstruction and highlighted its advantages over current methods. Direct integration with 
fluorescent microscopy and the possibility to use open microchannels could enable optomechanical cellular interrogations in the future.

\section{Experimental Section}

\section{Plant Models:}

Lilium longiflorum flowers are bought at a local florist shop. Individual stamens are placed in Eppendorf tubes and snap-frozen in liquid nitrogen. For long-term storage of pollen grains, the tubes are kept in a freezer at $-80{ }^{\circ} \mathrm{C}$. Before experiments, pollen are rehydrated by placing open Eppendorf tubes in a moist chamber at $24{ }^{\circ} \mathrm{C}$ for at least 30 minutes. The pollen grains are then submerged in growth medium to be injected into the PDMS device. The growth medium for lily pollen is prepared with the following components: $160 \mathrm{mM} \mathrm{H}_{3} \mathrm{BO}_{3}, 130 \mathrm{mM}$ $\mathrm{Ca}\left(\mathrm{NO}_{3}\right)_{2}, 1 \mathrm{mM} \mathrm{KNO}{ }_{3}, 5 \mathrm{mM} \mathrm{MES}$, and $10 \%$ sucrose. The rehydrated lily pollen grains are measured to have a major and minor axis of $112.5 \pm 8.9 \mu \mathrm{m}$ and $92.4 \pm 8.5 \mu \mathrm{m}$, respectively $(\mathrm{n}=40) .^{[31]}$

From fresh Arabidopsis thaliana plants, about 20 flowers are cut off and rehydrated in a moist chamber at room temperature. Subsequently, they are placed in an Eppendorf tube filled with growth medium. By shaking the Eppendorf tubes, the pollen grains are released and submerged in the liquid. The flowers, floating on the surface after centrifugation, are then manually removed. The growth medium for the Arabidopsis pollen is prepared with the following components: $1.6 \mathrm{mM} \mathrm{H}_{3} \mathrm{BO}_{3}, 5 \mathrm{mM} \mathrm{CaCl}_{2}, 5 \mathrm{mM} \mathrm{KCl}, 1 \mathrm{mM} \mathrm{MgSO}$, $10 \%$ sucrose, and $1 \%$ low melting agarose at $\mathrm{pH} 7.5$. The major and minor axis of the Arabidopsis pollen grains are found to be $26.7 \pm 2.4 \mu \mathrm{m}$ and $20.0 \pm 1.3 \mu \mathrm{m}$, respectively $(\mathrm{n}=40){ }^{[31]}$

Device Fabrication: The PDMS-based (Sylgard 184, Dow Corning, USA) microfluidic channels are fabricated using soft-lithography and mold replica techniques. The dimensions of 
the microchannel are $240 \mathrm{~mm}, 2 \mathrm{~mm}$ and $160 \mu \mathrm{m}$ in width, length and height, respectively. The PDMS structures are bonded onto a glass slide by oxygen plasma treatment. Subsequently, a piezo-electric transducer (KPEG-126, Kingstate, Taiwan) is fixed near the microchannel, as shown in the schematic of Figure 1 a), using epoxy. The transducer is excited at frequencies between $15-30 \mathrm{kHz}$ with an input power of 1-20 $\mathrm{V}_{\mathrm{PP}}$ via an arbitrary function generator (AFG3011C, Tektronix, USA). The microchannel contains a linear array of microcavities designed along one side of the channel. Each microcavity has a dimension of $60 \mu \mathrm{m}$ in width and $80 \mu \mathrm{m}$ in depth. When the growth medium of properties similar to water is injected, air bubbles are simultaneously trapped due to hydrophobic/ hydrophilic interactions within the microcavities. Additionally, it is possible to chemically bond the PDMS device up-side down (Figure S2) onto a glass slide, which creates an open microchannel (Figure 6). By placing liquid onto the channel region, the air bubbles get trapped in the cavities, similar to the closed channels. The device can be mounted on inverted microscopes for rotational characterization of plants cells. All bright field experiments are recorded at 13.3 - 15 frames per second, while 10 frames per seconds are used for fluorescent imaging (except for 3D reconstruction, see below). All videos provided in the supplementary material are real-time.

Fluorescent Labelling: For nuclei staining of Lilium longiflorum pollen grains, 4',6diamidino-2-phenylindole (DAPI, Sigma Aldrich, USA) is dissolved in DI water to achieve a final concentration of $2.5 \mu \mathrm{ml}^{-1}$. The pollen grains are immersed in the final solution and incubated for $10 \mathrm{~min}$ before injection into the microchannel. The fluorescent dye is excited and recorded at $358 \mathrm{~nm}$ and $461 \mathrm{~nm}$, respectively. The pollen tubes successfully germinate under the used concentration. 
3D Reconstruction: The pollen are stained using the protocol above. To prevent motion blur during recording, the rotational velocity of the pollen grain is minimized by driving the piezotransducer at a low single-digit voltage. The data is captured at a frame rate of 19 frames per second. The time lapse imaging stacks of the rotating pollen grain at all captured focal planes are then run through a series of image processing filters in MATLAB to denoise the frames and highlight features. This processing workflow includes rolling ball background subtraction, bilateral filtering ${ }^{[56]}$, image deconvolution, unsharp masking, and local Laplacian filtering ${ }^{[57]}$. The SIFT algorithm is then applied to the processed images to detect and describe local features, followed by feature matching and bundle adjustment ${ }^{[58]}$ for sparse reconstruction. The reconstruction is then further improved by running clustering views for multi-view stereo (CMVS) algorithms to obtain a dense 3D reconstruction ${ }^{[59]}$. This reconstruction procedure was performed using the Visual Structure from Motion package cite ${ }^{[60]}$

\section{Supporting Information}

Supporting Information is available from the Wiley Online Library or from the author.

\section{Acknowledgements}

The authors thank Jan Burri (ETH Zurich) and Chengzhi Hu (ETH Zurich) for the discussions. This work is supported by the ETH Zurich, the University of Zurich, and, in part, by a grant from SystemsX.ch, the Swiss Initiative in Systems Biology (Research and Technology Development Project MecanX) to U.G. and B.J.N. and an interdisciplinary grant from the Swiss National Science Foundation (Grant Number CR22I2_166110) to U.G. and B.J.N.. Daniel Ahmed also acknowledges the ETH Zurich Career Seed Grant-14 17-2.

Received: ((will be filled in by the editorial staff))

Revised: ((will be filled in by the editorial staff)) Published online: ((will be filled in by the editorial staff))

\section{References}

[1] S. Torino, M. Iodice, I. Rendina, G. Coppola, E. Schonbrun, Sensors 2016, 16, 1326.

[2] L. Huang, P. Zhao, W. Wang, Lab Chip 2018, DOI 10.1039/C8LC00407B.

[3] N. Pamme, Lab Chip 2006, 6, 24.

[4] P. W. Egolf, N. Shamsudhin, S. Pané, D. Vuarnoz, J. Pokki, A. G. Pawlowski, P. Tsague, B. De Marco, W. Bovy, S. Tucev, M. H. D. Ansari, B. J. Nelson, J. Appl. Phys. 2016, DOI 10.1063/1.4960406. 
[5] R. Dasgupta, S. Ahlawat, R. S. Verma, P. K. Gupta, Opt. Express 2011, 19, 7680.

[6] S. Orbay, A. Ozcelik, J. Lata, M. Kaynak, M. Wu, T. J. Huang, J. Micromechanics Microengineering 2017, 27, 15008.

[7] J. Nam, C. S. Lim, Sensors Actuators, B Chem. 2018, DOI 10.1016/j.snb.2017.09.173.

[8] P.-H. Huang, C. Y. Chan, P. Li, Y. Wang, N. Nama, H. Bachman, T. J. Huang, Lab Chip 2018, 18, 1411.

[9] M. V. Patel, I. A. Nanayakkara, M. G. Simon, A. P. Lee, Lab Chip 2014, 14, 3860.

[10] G. Destgeer, J. H. Jung, J. Park, H. Ahmed, K. Park, R. Ahmad, H. J. Sung, RSC Adv. 2017, 7, 22524.

[11] D. J. Collins, R. O’Rorke, C. Devendran, Z. Ma, J. Han, A. Neild, Y. Ai, Phys. Rev. Lett. 2018, DOI 10.1103/PhysRevLett.120.074502.

[12] V. H. Lieu, T. A. House, D. T. Schwartz, Anal. Chem. 2012, 84, 1963.

[13] F. Guo, Z. Mao, Y. Chen, Z. Xie, J. P. Lata, P. Li, L. Ren, J. Liu, J. Yang, M. Dao, S. Suresh, T. J. Huang, Proc. Natl. Acad. Sci. 2016, 113, 1522.

[14] P. Marmottant, S. Hilgenfeldt, Proc. Natl. Acad. Sci. 2004, 101, 9523.

[15] I. Leibacher, P. Hahn, J. Dual, Microfluid. Nanofluidics 2015, 19, 923.

[16] F. Guo, W. Zhou, P. Li, Z. Mao, N. H. Yennawar, J. B. French, T. J. Huang, Small 2015, DOI 10.1002/smll.201403262.

[17] P.-H. Huang, M. I. Lapsley, D. Ahmed, Y. Chen, L. Wang, T. J. Huang, Appl. Phys. Lett. 2012, 101, 141101.

[18] W. F. Fang, A. P. Lee, Microfluid. Nanofluidics 2015, DOI 10.1007/s10404-014-15255.

[19] S. Y. Tang, B. Ayan, N. Nama, Y. Bian, J. P. Lata, X. Guo, T. J. Huang, Small 2016, DOI 10.1002/smll.201600737.

[20] D. Ahmed, T. Baasch, N. Blondel, N. Läubli, J. Dual, B. J. Nelson, Nat. Commun. 2017, 8, DOI 10.1038/s41467-017-00845-5.

[21] D. Ahmed, M. Lu, A. Nourhani, P. E. Lammert, Z. Stratton, H. S. Muddana, V. H. Crespi, T. J. Huang, Sci. Rep. 2015, 5, 9744.

[22] D. Ahmed, T. Baasch, B. Jang, S. Pane, J. Dual, B. J. Nelson, Nano Lett. 2016, 16, 4968.

[23] M. Kaynak, A. Ozcelik, A. Nourhani, P. E. Lammert, V. H. Crespi, T. J. Huang, Lab Chip 2017, 17, 395.

[24] T. Hayakawa, S. Sakuma, F. Arai, Microsystems Nanoeng. 2015, 1, 15001.

[25] A. Ozcelik, N. Nama, P.-H. Huang, M. Kaynak, M. R. McReynolds, W. Hanna-Rose, T. J. Huang, Small 2016, 12, 5120.

[26] C. Wang, B. Rallabandi, S. Hilgenfeldt, Phys. Fluids 2013, 25, 22002.

[27] D. Ahmed, A. Ozcelik, N. Bojanala, N. Nama, A. Upadhyay, Y. Chen, W. HannaRose, T. J. Huang, Nat. Commun. 2016, 7, 11085.

[28] J. Dumais, J. Exp. Bot. 2013, 64, 4684.

[29] A. F. Edlund, Q. Zheng, N. Lowe, S. Kuseryk, K. L. Ainsworth, R. H. Lyles, S. J. Sibener, D. Preuss, Am. J. Bot. 2016, 103, 1006.

[30] N. Läubli, N. Shamsudhin, D. Ahmed, B. J. Nelson, in Procedia CIRP, 2017, pp. 9398.

[31] N. Shamsudhin, N. Laeubli, H. B. Atakan, H. Vogler, C. Hu, W. Haeberle, A. Sebastian, U. Grossniklaus, B. J. Nelson, PLoS One 2016, 11, e0168138.

[32] M. M. Wudick, M. T. Portes, E. Michard, P. Rosas-Santiago, M. A. Lizzio, C. O. Nunes, C. Campos, D. Santa Cruz Damineli, J. C. Carvalho, P. T. Lima, O. Pantoja, J. A. Feijó, Science (80-. ). 2018, 360, 533.

[33] J. T. Burri, H. Vogler, N. F. Läubli, C. Hu, U. Grossniklaus, B. J. Nelson, New Phytol. 2018, DOI 10.1111/nph.15260.

[34] J. Zhang, Q. Huang, S. Zhong, A. Bleckmann, J. Huang, X. Guo, Q. Lin, H. Gu, J. 
Dong, T. Dresselhaus, L.-J. Qu, Nat. Plants 2017, 3, 17079.

[35] E. Katifori, S. Alben, E. Cerda, D. R. Nelson, J. Dumais, Proc. Natl. Acad. Sci. U. S. A. 2010, 107, 7635.

[36] A. R. Tovar, M. V. Patel, A. P. Lee, Microfluid. Nanofluidics 2011, 10, 1269.

[37] Y. Okabe, A. P. Lee, J. Lab. Autom. 2014, 19, 163.

[38] M. V. Patel, A. R. Tovar, A. P. Lee, Lab Chip 2012, 12, 139.

[39] B. Rallabandi, A. Marin, M. Rossi, C. Kähler, S. Hilgenfeldt, J. Fluid Mech. 2015, 777, 408.

[40] R. Manasseh, P. Tho, A. Ooi, K. Petkovic-Duran, Y. Zhu, Phys. Procedia 2010, 3, 427.

[41] P. Song, X. Dong, X. Liu, Biomicrofluidics 2016, 10, 011912.

[42] J. Park, J. H. Jung, G. Destgeer, H. Ahmed, K. Park, H. J. Sung, Lab Chip 2017, DOI 10.1039/C6LC01405D.

[43] P. Marmottant, S. Hilgenfeldt, Nature 2003, 423, 153.

[44] O. Sawatzki, Acta Mech. 1970, 9, 159.

[45] J. Park, D. Kurihara, T. Higashiyama, H. Arata, Sensors Actuators, B Chem. 2014, 191, 178.

[46] K. Gooh, M. Ueda, K. Aruga, J. Park, H. Arata, T. Higashiyama, D. Kurihara, Dev. Cell 2015, 34, 242.

[47] S. S. Gorthi, D. Schaak, E. Schonbrun, Opt. Express 2013, 21, 5164.

[48] H. Halbritter, Biotech. Histochem. 1998, 73, 137.

[49] C. G. Jones, in Forensic Microsc. Skelet. Tissues, 2012, pp. 1-20.

[50] H. Vogler, D. Felekis, B. Nelson, U. Grossniklaus, Plants 2015, 4, 167.

[51] J. T. Burri, C. Hu, N. Shamsudhin, X. Wang, H. Vogler, U. Grossniklaus, B. J. Nelson, IEEE Int. Conf. Autom. Sci. Eng. 2016, 2016-Novem, 942.

[52] E. Kranz, H. Lörz, Sex. Plant Reprod. 1990, 3, DOI 10.1007/bf00205225.

[53] H. Qu, W. Xing, F. Wu, Y. Wang, PLoS One 2016, 11, e0152320.

[54] H. Feng, G. Barbosa-Canovas, J. Weiss, Eds., Ultrasound Technologies for Food and Bioprocessing, Springer New York, 2011.

[55] L. A. Crum, J. Acoust. Soc. Am. 1980, 68, 203.

[56] S. Paris, S. W. Hasinoff, J. Kautz, Acm Siggraph 2011 2015, DOI 10.1145/2723694.

[57] S. Paris, P. Kornprobst, J. Tumblin, Bilateral Filtering, Now Publishers Inc., Hanover, MA, USA, 2009.

[58] C. Wu, URL http//www. cs. washington. edu/homes/ccwu/vsfm 2016.

[59] Y. Furukawa, B. Curless, S. M. Seitz, R. Szeliski, in Proc. IEEE Comput. Soc. Conf. Comput. Vis. Pattern Recognit., 2010.

[60] C. Wu, S. Agarwal, B. Curless, S. M. Seitz, in Proc. IEEE Comput. Soc. Conf. Comput. Vis. Pattern Recognit., 2011. 


\section{Figures}

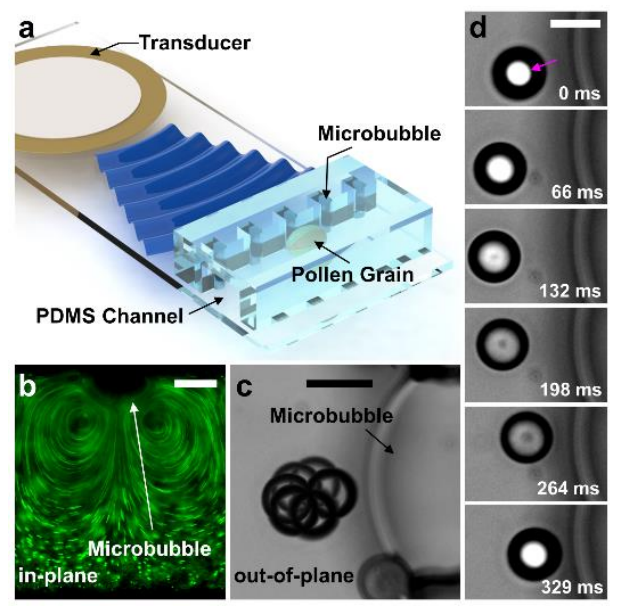

Figure 1. A schematic showing the experimental setup, and the visualization of vortices using microbeads. (a) The setup consists of an acoustic wave generating piezo-transducer, glued onto a glass slide adjacent to the PDMS channel. Air bubbles trapped along the channel generate vortices in the adjacent fluid due to their vibration, leading to the rotation of individual plant cells. (b) $1 \mu \mathrm{m}$ fluorescent particles showing a pair of counter-rotating inplane vortices symmetric to the microbubble. (c) A $10 \mu \mathrm{m}$ polystyrene particle moving along an out-of-plane vortex in front of a microbubble. (d) The image sequence shows the out-ofplane movement of the $10 \mu \mathrm{m}$ polystyrene particle from the previous subfigure. Its $3 \mathrm{D}$ movement is represented by the change in and out of focus, which leads to a significant change of the area highlighted by the magenta arrow. Scale bars: $b=50 \mu \mathrm{m}, \mathrm{c}=20 \mu \mathrm{m}, \mathrm{d}=$ $10 \mu \mathrm{m}$. 


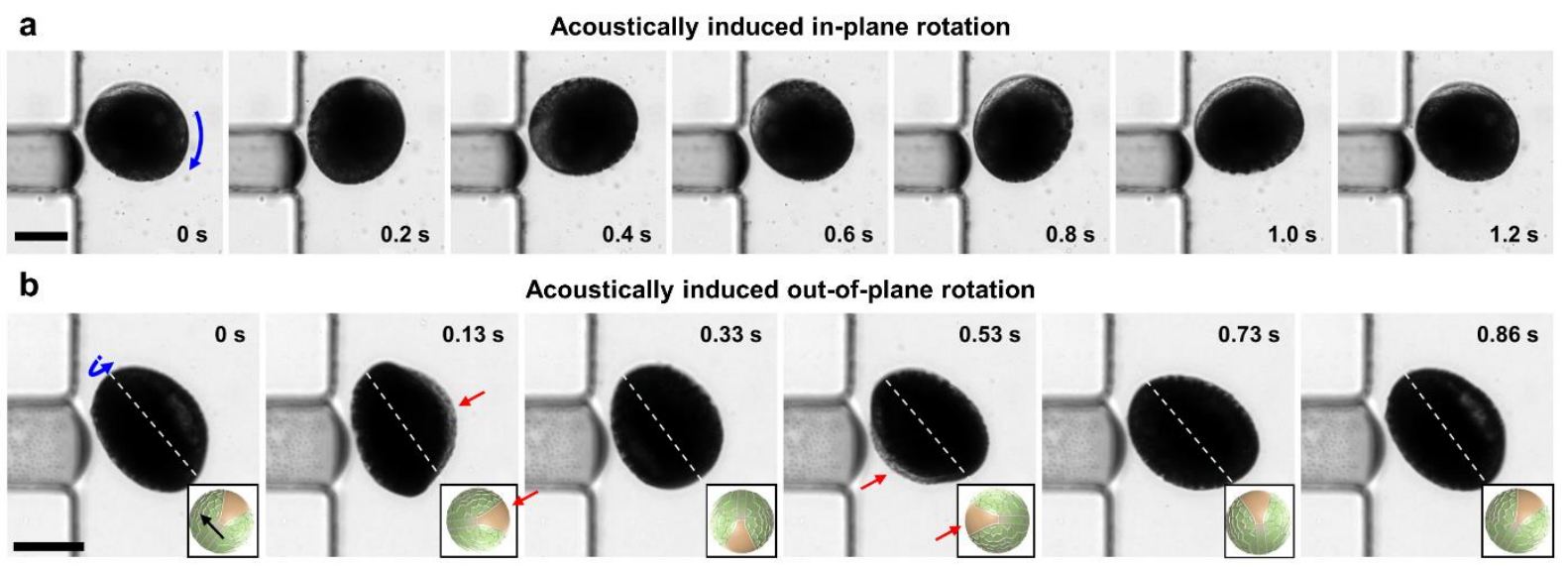

Figure 2. Optical images showing the rotation of lily pollen grains. (a) A sequence of optical images presenting the clockwise in-plane rotation of a lily pollen grain at a voltage and excitation frequency of $10 \mathrm{~V}_{\mathrm{PP}}$ and $24.1 \mathrm{kHz}$, respectively. (b) A lily pollen grain rotating outof-plane. The insets in the right corner illustrate the position of the colpus (marked with a red arrow if visible), if the pollen grain is observed along the direction of the black arrow. Scale bars $=50 \mu \mathrm{m}$. 


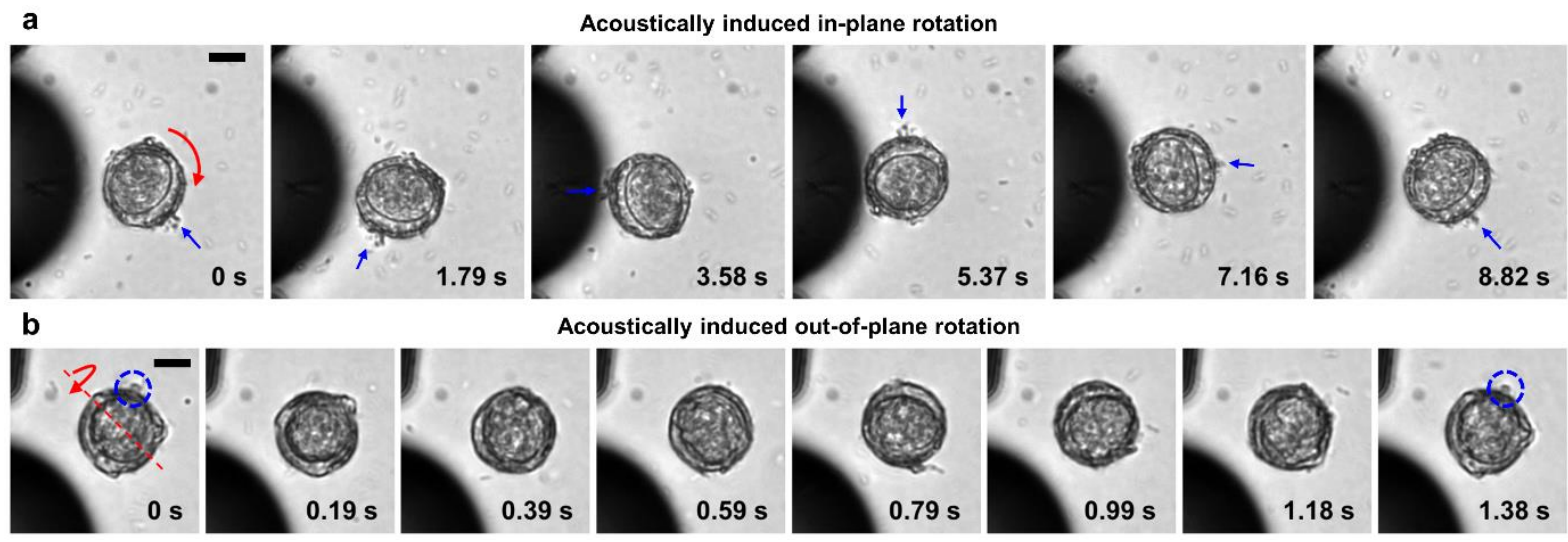

Figure 3. Microscope images visualizing the 3D rotation of Arabidopsis pollen grains. (a) A sequence showing the clockwise in-plane rotation of an Arabidopsis pollen grain near the microbubble (black area on the left side). The bubble is excited at $22.4 \mathrm{kHz}$ with a voltage of $1 \mathrm{VPP}$. (b) Optical images presenting a stable out-of-plane rotation of an Arabidopsis pollen grain using an excitation signal of $2 \mathrm{~V}_{\mathrm{PP}}$ at a frequency of $20 \mathrm{kHz}$. Parts of the microbubble can be seen in the bottom left corner. Scale bars $=10 \mu \mathrm{m}$. 

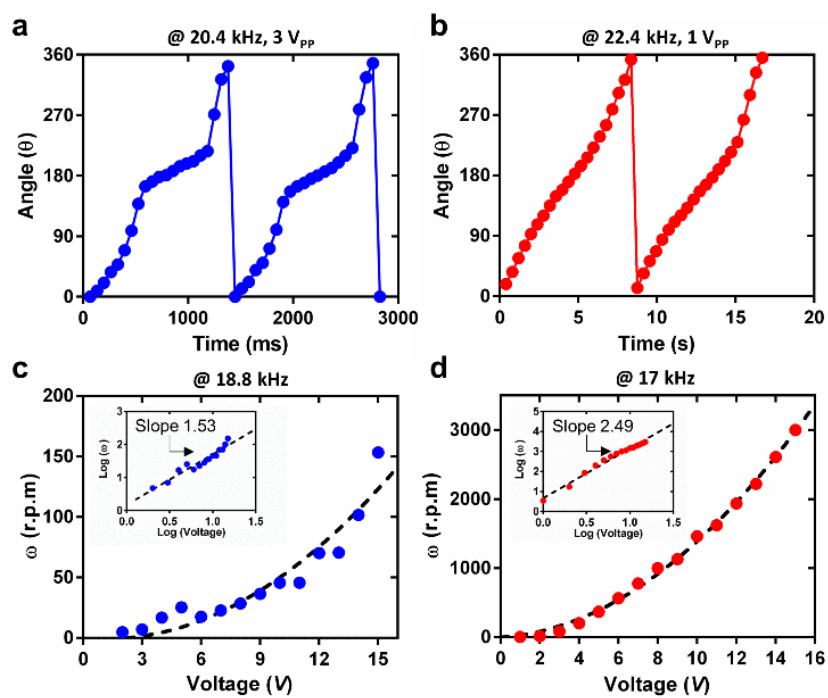

Figure 4. Analysis of the acoustically induced rotation of pollen grains with different dimensions. (a) In-plane rotation (two times $360^{\circ}$ ) of a lily pollen grain. The non-linearity observed arises from the shape anisotropy of the pollen grain. (b) In-plane rotation (two times $360^{\circ}$ ) of an Arabidopsis pollen grain. Compared to the lily pollen grain, the rotation shows a more linear behavior. (c) and (d) Angular velocity measured as a function of changing actuator voltage for lily and Arabidopsis pollen grain respectively. The insets show a exponential fitting factor of 1.53 for lily and 2.49 for Arabidopsis. 


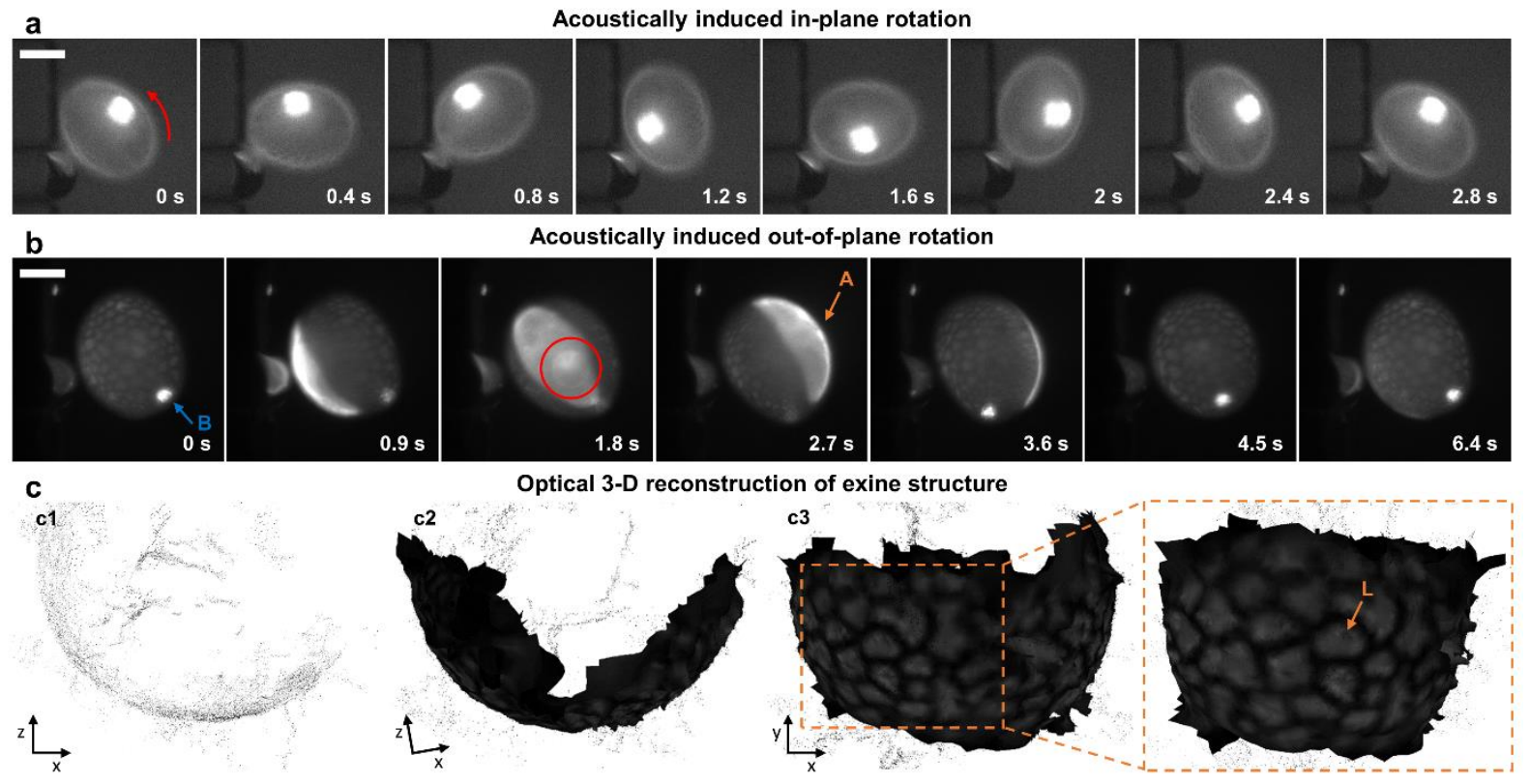

Figure 5. Optical images of fluorescently labeled lily pollen and an optical 3D reconstruction. (a) A lily pollen grain with a fluorescently labeled nucleus rotating in-plane. (b) A fluorescently labeled lily pollen grain performing a stable out-of-plane rotation. Depending on the orientation of the pollen grain, the auto-fluorescent colpus (A) partially obstructs the view onto intracellular features. The bright spot (B) is the vegetative nucleus, while the generative nucleus can be seen through the colpus at 1.8 seconds (red circle). (c) High resolution optical 3D reconstruction of the exine features of a fluorescent lily pollen grain. (c1) shows the dense point cloud used for processing, while (c2) includes the matched images and nicely presents the azimuthal angle of the reconstruction. (c3) shows a perpendicular view onto a part of the high-resolution reconstruction with the differently shaped lumina (L). Scale bars $=50 \mu \mathrm{m}$. 
a

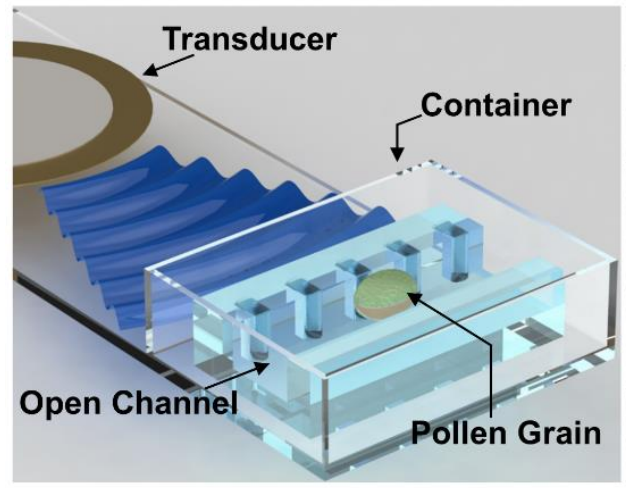

b

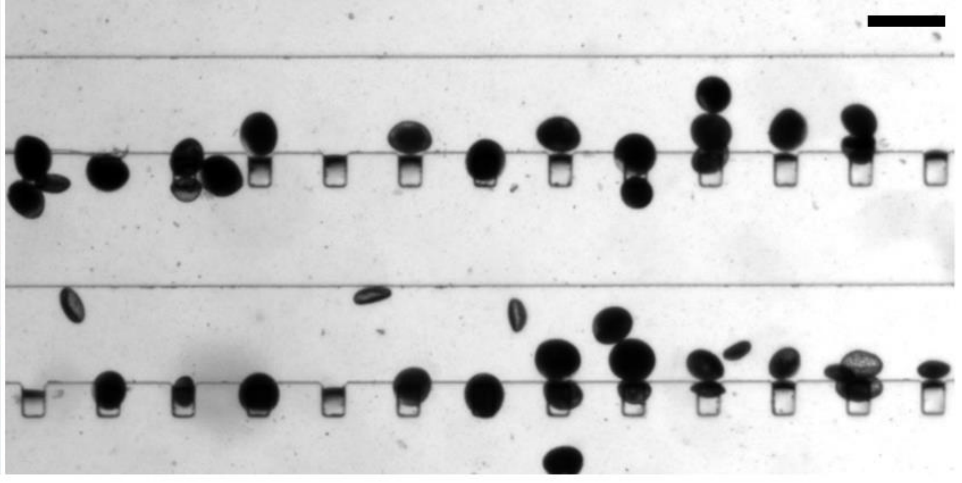

C

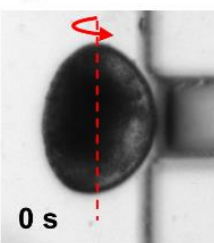

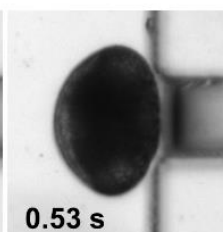

$0.53 \mathrm{~s}$

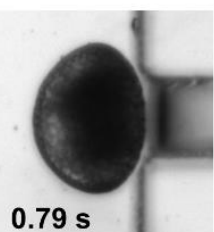

$0.79 \mathrm{~s}$
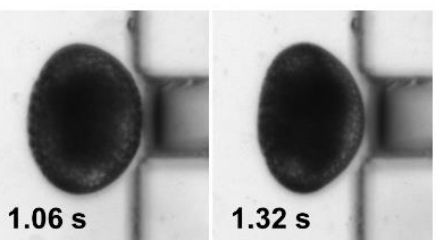

Figure 6. An open-microchannel setup and optical images of lily pollen grains rotating in an open channel. (a) A schematic showing the setup for manipulation of pollen grains in an open microchannel. To prevent leakage, the channel is encased with sidewalls while the top is open to enable direct access to the sample. (b) Two parallel open microfluidic channels with acoustically manipulated Lily pollen grains. Pollen grains can be trapped in front as well as above the microbubbles. (c) A series of optical images visualizing the slow and stable threedimensional rotation of a lily pollen grain in an open microchannel. The applied frequency and voltage are $27 \mathrm{kHz}$ and $7 \mathrm{~V}_{\mathrm{PP}}$, respectively. Scale bars: $\mathrm{b}=200 \mu \mathrm{m}, \mathrm{c}=40 \mu \mathrm{m}$. 